\title{
Sífilis na mulher e no homem: uma revisão integrativa das publicações científicas
}

\section{Syphilis in women and men: an integrative review of scientific publications}

Sifilis en la mujer y en el hombre: una revisión integrada de las publicaciones científicas

\author{
Leila Rangel da Silva'; Mirian Santos Paival'; Isis Vanessa Nazareth"l'; \\ Maíra Domingues Bernardes Silvalv; Eliza Cristina Macedo ${ }^{v}$
}

\begin{abstract}
RESUMO
Objetivo: revisar as publicações científicas sobre a sífilis na mulher e no homem e analisá-las de acordo com os níveis de evidência científica. Método: pesquisa bibliográfica, tipo revisão integrativa, realizada em abril de 2016. Coleta de informações nas bases de dados LILACS, MEDLINE, SciELO, Cochrane Library, Embase, CINAHL e na Scopus, da editora Elsevier. Resultado: encontraram-se 23 artigos, conforme os critérios de inclusão, publicados no período de 1990 a 2016 . O Brasil contribuiu com $12(52,1 \%)$ publicações, $13(56,52 \%)$ artigos estão relacionados a saúde materno-infantil e somente $1(4,3 \%)$ produção explana a saúde do homem no cuidado com a sífilis. Verificou-se que $21(91,3 \%)$ estudos são descritivos, classificados no nível de evidência científica 2C. Conclusão: os artigos foram classificados no nível 2C, considerado evidência científica moderada. A sífilis é muito explorada em assuntos que abrangem área materno-infantil, provocando um hiato de conhecimentos científicos voltados para a temática sífilis fora do ciclo gravídico-puerperal e no homem.

Descritores: Doenças sexualmente transmissíveis; sífilis; saúde da mulher; saúde do homem.
\end{abstract}

\section{ABSTRACT}

Objective: review scientific publications on syphilis in women and men and analyze them by level of scientific evidence. Method: for this integrative literature review conducted in April 2016, information was collected from the LILACS, MEDLINE, SciELO, Cochrane Library, Embase, CINAHL and Elsevier Scopus databases. Result: 23 articles, published between 1990 and 2016, were found to match the inclusion criteria. Brazil contributed 12 (52.1\%) publications, $13(56.52 \%)$ articles related to maternal and child health, and only $1(4.3 \%)$ addressed men's health in care for syphilis. $21(91.3 \%)$ of the studies were descriptive, and classified as at scientific evidence level 2C. Conclusion: the articles were classified as level $2 \mathrm{C}$, which reflects moderate scientific evidence. Syphilis is widely explored in topics addressing maternal and child care, but a gap exists in syphilis-related scientific knowledge outside the pregnancy-puerperal cycle and in men.

Descriptors: Sexually transmitted diseases; syphilis; women's health; men's health.

\section{RESUMEN}

Objetivo: revisar las producciones científicas publicadas sobre la sífilis en mujeres y hombres y analizarlas de acuerdo con los niveles de evidencia científica. Método: investigación bibliográfica, tipo de revisión integradora, realizada en abril de 2016. Recolección de información en las bases de datos LILACS, MEDLINE, SciELO, Cochrane Library, Embase, CINAHL y Scopus, editorial Elsevier. Resultado: se encontraron 23 artículos según los criterios de inclusión publicados entre 1990 y 2016 . Brasil contribuyó con $12(52,1 \%)$ publicaciones, $13(56.52 \%)$ artículos se relacionaron con la salud materna e infantil y apenas una (4.3\%) producción explica la salud de los hombres en la atención respecto a la sífilis. Se encontró que 21(91.3\%) estudios son descriptivos, clasificados dentro del nivel de evidencia científica 2C. Conclusión: se clasificaron los artículos en el nivel 2C, considerados evidencia científica moderada. La sífilis se explora ampliamente en temas que cubren el área materna e infantil, lo que causa una brecha de conocimiento científico volcado hacia el tema de la sífilis fuera del ciclo del embarazo/puerperio y el hombre.

Descriptores: Enfermedades de transmisión sexual; sífilis; salud de la mujer; salud del hombre.

\section{INTRODUÇÃO}

A alta incidência de infecções sexualmente transmissíveis tem se tornado grande problema de saúde pública. Vale refletir sobre as constantes descobertas e avanços na área da saúde, e, no que concerne ao controle dessas infecções, não é necessária sofisticação tecnológica para ações de prevenção e tratamento, principalmente quando se aborda a sífilis, infecção que historicamente ocasiona fortes críticas sociais ${ }^{1,2}$. No período de 2010 a junho de 2016, foram notificados 227.663 casos de sífilis adquirida no Brasil $^{3}$.

'Enfermeira. Pós-Doutora. Professora Associada, Universidade Federal do Estado do Rio de Janeiro. Brasil. E-mail: rangel.leila@gmail.com "Enfermeira. Pós-Doutora. Professora Associada, Universidade Federal da Bahia. Brasil. E-mail: paivamirian@hotmail.com I'Enfermeira. Doutora. Professora Adjunta, Universidade Federal do Rio de Janeiro. Brasil. E-mail: ivnenfermagem@gmail.com

IVEnfermeira. Mestre. Tecnologista em Saúde Pública, Instituto Fernandes Figueira. Rio de Janeiro, Brasil. E-mail: mairinhadbs@gmail.com

vEnfermeira. Doutora. Professora Adjunta, Universidade Federal do Estado do Rio de Janeiro. Brasil. E-mail: macedo.unirio@gmail.com 
Ao se reconhecer a significação do papel de gênero em permanente reestruturação em relação à mulher e ao homem, há necessidade de reflexão e intervenção, por parte dos profissionais de saúde, no que diz respeito ao cuidado prestado à mulher e ao homem com sífilis. Identificar a produção científica sobre a sífilis relacionada à saúde feminina e masculina é fundamental para dar visibilidade às pesquisas já realizadas ${ }^{4}$.

Nesse panorama surge o seguinte questionamento: o que refletem as publicações científicas sobre sífilis na mulher e no homem?

Diante dessa pergunta, objetivou-se revisar as publicações científicas sobre a sífilis na mulher e no homem e analisá-las de acordo com os níveis de evidência científica.

\section{METOdOLOGIA}

Pesquisa bibliográfica, descritiva, baseada na revisão integrativa. Este tipo de estudo possibilita sintetizar as diferentes pesquisas disponíveis sobre determinado assunto e orienta a prática com embasamento no conhecimento científico. A revisão integrativa é composta por seis fases: estabelecimento da questão de pesquisa; busca na literatura; categorização dos estudos; avaliação dos estudos incluídos; interpretação dos resultados e síntese do conhecimento ${ }^{5}$.

As investigações encontradas nesta revisão integrativa foram classificadas segundo a Prática Baseada em Evidências (PBE). Esta classificação está em conformidade com Oxford Centre Evidence-Based Medicine (Figura 1) que classifica as evidências conforme o delineamento metodológico ${ }^{6}$. Ver Figura 1.

\begin{tabular}{ll}
\hline $\begin{array}{l}\text { Nível de } \\
\text { evidência }\end{array}$ & Tipos de estudo \\
\hline 1A & $\begin{array}{l}\text { Revisões sistemáticas e metanálises de ensaios clínicos comparáveis. Estudos controlados randomizados bem } \\
\text { delineados com desfecho clínico relevante. }\end{array}$ \\
1B & Estudos controlados randomizados com estreito intervalo de confiança. \\
1C & Resultados do tipo tudo ou nada. Estudo de série de casos controlados. \\
2A & Revisão sistemática homogênea de estudos de coorte (com grupos de comparação e controle de variáveis). \\
2B & Estudo de coorte com pobre qualidade de randomização, controle ou sem acompanhamento longo, estudo \\
2C & de coorte transversal. \\
3A & Resultados de pesquisas (observação de resultados terapêuticos ou evolução clínica). \\
3B & Revisão sistemática homogênea de estudos de caso com grupo-controle. \\
4 & Estudos de caso com grupo-controle. \\
5 & Relatos de caso e série sem definição de caso controle. \\
\hline
\end{tabular}

FIGURA 1: Classificação dos tipos de estudo por nível de evidência.

O ajuizamento dos achados científicos relacionados ao tema proposto foi realizado por duas pesquisadoras independentes. Os critérios de inclusão foram artigos científicos originais nacionais e internacionais, publicados em Português, Inglês ou Espanhol, independente do método de pesquisa utilizado. Estudos recuperados em mais de uma base de dados foram considerados somente uma vez. Foram excluídos registros que não comtemplaram o homem ou a mulher como objeto do cuidado, bem como editoriais, resumos de anais, relatórios de gestão e boletins epidemiológicos.

Como estratégia de busca para sífilis relacionada à saúde da mulher e do homem, utilizaram-se certos Descritores em Ciências da Saúde (DeCS) do Portal Biblioteca Virtual de Saúde (BVS), com base na interface de pesquisa avançada via formulário $i A H$, aplicando-se os operadores booleanos or/and. Os descritores selecionados foram: sífilis; sorodiagnóstico da sífilis; assistência integral à saúde da mulher; saúde do homem.

A obtenção da produção científica aconteceu por busca bibliográfica nas bases de dados da BVS - Literatura LatinoAmericana e do Caribe em Ciências da Saúde (LILACS); Scientific Electronic Library Online (SciELO); The Cochrane Library; Literatura Internacional em Ciências da Saúde (MEDLINE); ainda, foram acessadas as bases de dados Embase, Cumulative Index of Nursing and Allied Health (CINAHL) e Scopus, da editora Elsevier, durante o mês de abril de 2016.0 intervalo temporal não foi predeterminado com o intuito de apurar, nas bases de dados, o início da produção científica e difusão da temática em questão. 
Para a extração e caracterização da amostra, foi elaborada uma ficha de análise documental obtida através do modelo de dois estudos ${ }^{7,8}$, composta pelas seguintes variáveis: ano de publicação, procedência da produção, área do conhecimento; objetivo da investigação; participantes do estudo; delineamento da pesquisa; nível de evidência e periódicos.

Na síntese dos dados foi realizada a estatística descritiva apresentada na forma de frequência absoluta e relativa. Os estudos foram divididos em subgrupos, em conformidade com a classificação estabelecida na ficha documental. A categorização foi baseada no tipo de incidência e características da amostra.

A partir da busca foram encontrados 148 estudos, sendo 30(20,2\%) publicados na LILACS, 11(7,4\%) na SciELO, 49(33,1\%) na MEDLINE, 6(4,05\%) na Cochrane Library, 16(10,8\%) na Embase, 15(10,1\%) na CINAHL e 21(14,1\%) na Scopus.

Os 148 artigos foram submetidos, por sua vez, a leitura minuciosa dos títulos e resumos. Destes, somente 99 eram artigos científicos originais, publicados na íntegra em Português, Inglês ou Espanhol. Fez-se, então, a leitura das 99 publicações, das quais $27(27 \%)$ se encontram na LILACS; $2(2 \%)$ na SciELO e $14(14 \%)$ na MEDLINE, 2(2\%) na Cochrane Library, 19(19\%) na Embase, 20(20\%) na CINAHL e 15(15\%) na Scopus, desse quantitativo, apenas 23 se adequaram a todos os critérios de inclusão. Vale destacar que seis publicações da LILACS também se encontravam na SciELO e, nove publicações da CINAHL encontravam-se na Scopus e, pela duplicidade, não foram consideradas.

\section{RESULTADOS}

Pontua-se que os 23 (100\%) artigos identificados ${ }^{9-31}$ foram publicados entre os anos de 1990 a 2016, ressaltando que $16(69,3 \%)$ investigações foram elaboradas pela área médica ${ }^{10-15,17,20-25,27,29,31}$.

Com relação à variável procedência da produção, observa-se, a distribuição das pesquisas acerca da sífilis em nível mundial, na Tabela 1.

\begin{tabular}{lcr}
\multicolumn{3}{l}{ TABELA 1: Distribuição das publicações sobre sífilis } \\
por área de procedência. Rio de Janeiro, Brasil, 2016 \\
\hline Procedência & $\mathbf{f}$ & $\%$ \\
\hline Brasil - Rio de Janeiro & 8 & 34,9 \\
Brasil- São Paulo & 2 & 8,9 \\
Brasil- Bahia & 1 & 4,3 \\
Brasil-Porto Alegre & 1 & 4,3 \\
Uruguai - Montevidéu & 1 & 4,3 \\
Argentina & 1 & 4,3 \\
Venezuela & 1 & 4,3 \\
Republica Dominicana- Haiti & 1 & 4,3 \\
República Checa - Praga & 1 & 4,3 \\
Estados Unidos & 3 & 13,2 \\
China & 1 & 4,3 \\
Moçambique- Maputo & 1 & 4,3 \\
Peru-Lima & 1 & 4,3 \\
Total & 23 & 100,0 \\
\hline
\end{tabular}

A maioria - 12(52,1\%) - procede do Brasil, seguindo os Estados Unidos com 3(13,2\%) e os demais países com apenas uma produção cada. Ver Tabela 1.

A distribuição de publicações sobre sífilis por área do conhecimento é especificada na Tabela 2.

A maioria das pesquisas revisadas procede da medicina e a enfermagem é representada em 3(13,2\%) publicações, segundo a Tabela 2.

A respeito dos objetivos dos estudos, $7(30,43 \%)$ abordaram diagnóstico e tratamento da sífilis em mães e recémnascidos ${ }^{9,11,16,18-21}$ e $6(26,08 \%)$ buscaram avaliar a efetividade de programas e campanhas para erradicação da sífilis congênita no intuito de contribuir com a reorientação da intervenção sobre saúde da população de gestantes e seus filhos $^{10-15}$. 
TABELA 2: Distribuição da quantidade de publicações sobre sífilis por área do conhecimento. Rio de Janeiro, RJ, Brasil, 2016

\begin{tabular}{llr}
\hline Área profissional & $\mathbf{f}$ & \multicolumn{1}{c}{$\%$} \\
\hline Medicina & 16 & 69,3 \\
Enfermagem & 3 & 13,2 \\
Ciências Biológicas & 3 & 13,2 \\
Antropologia & 1 & 4,3 \\
Total & 23 & 100,0 \\
\hline
\end{tabular}

A correlação entre a prevalência de infecções sexualmente transmissíveis com os dados epidemiológicos, sífilis e grupos vulneráveis (aqueles envolvidos com drogas ilícitas, álcool, privados de liberdade, profissionais do sexo e moradores de rua) foram encontrados em 6(26,08\%) produções $22-26,31$.

Relacionar a sífilis com outras infeç̧ões por transmissão sexual, como a gonorreia, Hepatite B e o Vírus da Imunodeficiência Humana (HIV), foi o objetivo de 4(17,4\%) artigos ${ }^{25-27,30}$. Assinala-se um único estudo cujo objetivo foi discorrer sobre a sexualidade masculina e a sífilis, a partir do contraponto de moradores em situação de rua ${ }^{31}$.

Dos 23 estudos, 11(47,82\%) coletaram dados pelo meio de entrevistas ou questionários ${ }^{9,10,16-18,20-25}$, sendo que, destes, 6(26,08\%) envolveram mulheres que possuem filhos recém-nascidos ${ }^{9,10,16,18-20}$ e $6(26,08 \%)$ visaram população em situação de vulnerabilidade ${ }^{22-26,31}$. Os $6(26,08 \%)$ estudos que tiveram como participantes as mulheres e recémnascidos levaram em consideração variáveis socioeconômicas maternas s,10,16,18-20 $^{\text {. }}$

Quanto ao delineamento das pesquisas, verificou-se que 21(91,3\%) estudos foram descritivos (não experimentais) com observação de resultados terapêuticos e evolução clínica ${ }^{9-18,20,22-31}$. Houve $1(4,3 \%)$ artigo baseado no método de estudo de caso afim de documentar e interpretar o adoecimento por sífilis em contexto particular ${ }^{21}$. A metodologia da revisão integrativa de literatura foi utilizada em apenas $1(4,3 \%)$ publicação ${ }^{19}$.

Dessa forma, verifica-se que $21(91,3 \%)$ publicações foram classificadas no nível de evidencia 2( $C^{9-18,20,22-31}$; os demais artigos condizem com o nível evidencia $4^{21}$ e $5^{19}$.

No que tange ao enfoque da produção, é possível notar aspectos baseados na clínica e na epidemiologia $11(47,82 \%)^{10,20,22-29,31}$, seguidos pela perspectiva da avaliação de serviço $-4(17,3 \%)^{12-15}$, diagnóstico e tratamento $4(17,3 \%)^{9,11,19,30}$, abordagem sociocultural e histórica - 4(17,3\%) $16-18,21$ - neste último, 2(8,9\%) artigos incluem o olhar do cuidado ${ }^{16,18}$.

Constata-se que os periódicos que publicaram a maior produção de artigos associados à sífilis na saúde da mulher e do homem foram a Revista Brasileira de Epidemiologia - 3(13,2 $)^{13,30,31}$; a Revista de Saúde Pública - 2(8,9\% $)^{9,24}$ e o Caderno de Saúde Pública, também com $2(8,9 \%)^{12,15}$.

\section{DISCUSSÃO}

Ao analisar a variável ano de publicação, para o esclarecimento do início da produção científica e difusão da temática em questão, averiguou-se grande alternância entre os anos de 1990 a 2016. Vale destacar que a primeira publicação on-line, na íntegra, intitulada: Sorologia positiva para sífilis, toxoplasmose e doença de chagas em gestantes de primeira consulta em centros de saúde de área metropolitana - Brasil, data de $1990^{9}$, dois anos antes de o Ministério da Saúde consolidar três ações importantes visando o combate da sífilis: atribuição ao Sistema de Informação e Agravos (SINAN) a notificação compulsória dos casos confirmados de infecções sexualmente transmissíveis; a publicação do documento intitulado: Bases técnicas para a eliminação da sífilis congênita; e a assinatura do Acordo Internacional para a Eliminação da Sífilis Congênita nas Américas ${ }^{3,32-34}$.

Com referência à procedência da produção, o estado brasileiro que publicou o maior número de estudos foi o Rio de Janeiro - 8(34,9\% $)^{10-15,18,21}$, seguido por São Paulo - 2(8,9\% ${ }^{23,31}$, como mostra a Tabela 1. Essa quantidade remete à concentração, na Região Sudeste, dos centros de pesquisa e de pós-graduação, já que dos 1.420 programas e cursos de pós-graduação, $844(53,4 \%)$ encontram-se nessa região, considerada o polo de geração de conhecimento no país ${ }^{32,34}$.

É importante destacar que, entre todos os países que publicaram estudos sobre a sífilis na população feminina e masculina, o Brasil contribuiu com a maior produção - 12(52,1\%) ${ }^{9-16,21,23,30,31}$. Salienta-se que, além de publicações advindas dos estados do Rio de Janeiro ${ }^{10-15,18,21}$ e São Paulo ${ }^{23,31}$, pesquisas também procederam da Bahia ${ }^{16}$ e Porto 
Alegre $^{30}$, colaborando com a divulgação e ampliação do conhecimento necessário para subsidiar a eliminação da sífilis. Em seguida, destacam-se 3(13,2\% $)^{22,27,29}$ produções dos Estados Unidos, conforme evidencia a Tabela 1.

No tocante à área de conhecimento, verificou-se que $16(69,3 \%)$ estudos publicados relativos à sífilis na saúde da mulher e do homem procedem da Medicina ${ }^{10-15,20-25,27,29-31}$, como apresenta a Tabela 2. Esse resultado pode ser explicado pelo fato de os grupos de pesquisa da área médica contarem com os maiores incentivos financeiros voltados para a produção de conhecimento, principalmente no aspecto clínico e epidemiológico.

Os demais se distribuíram através da enfermagem - 3(13,2\% $)^{9,16,18}$ e ciências biológicas - 3(13,2\% $)^{19,26,28}$, seguido pela Antropologia - 1(4,3\% $)^{17}$, segundo a Tabela 2.

Cabe destacar que a parceria entre os profissionais de saúde e de outras áreas externas à saúde é relevante, com troca de experiência e colaboração para a produção científica, com intuito de contribuir com a qualidade da assistência à mulher e ao homem com sífilis. Sabe-se que a compreensão dos problemas de saúde requer abordagem ecológica dos problemas para formulação de políticas públicas ${ }^{32,34}$.

Com base na variável objetivos da investigação, certificou-se que $13(56,52 \%)$ estudos atentaram para as lacunas existentes na saúde materno-infantil, inclusive houve a preocupação com os efeitos das ações e práticas de saúde implementadas pelos programas de combate a sífilis congênita e neonatal ${ }^{9-16,18-21,30}$

Os resultados incitam discussões de cunho assistencial com apontamentos para a necessidade de qualificação do pré-natal e capacitação continuada dos profissionais de saúde. Estimulam, do mesmo modo, aspectos operacionais do sistema de diagnóstico que deve ser eficiente, pois o custo do exame laboratorial para detecção da infecção é baixo e a tecnologia é simples. Ademais, exortam demandas para atuação da vigilância epidemiológica com intuito de dimensionar uma visão global das necessidades para ações eficientes dos programas de saúde que envolve a eliminação da sífilis ${ }^{9-16,18-21,30}$.

Dar visibilidade à sífilis na área da saúde materno-infantil, como observado por meio da expressividade desta temática nos objetivos das pesquisas, é refletir sobre a importância da intervenção necessária e oportuna para sua erradicação, uma vez que o período determinado para a eliminação desta infecção esgotou-se em 2015 2,9-16,18-21,30,33.

No que se refere aos demais estudos, 6(26,08\%) deles objetivaram a análise epidemiológica da sífilis em grupos vulneráveis relacionados ao tráfico e uso de drogas ilícitas, álcool, prostituição ou privação de liberdade ${ }^{22-26,31}$. Esse delineamento colabora para a avaliação de questões de saúde alusivas ao sistema prisional, as profissionais do sexo, e também aos indivíduos envolvidos com drogas ilícitas e com o álcool. Contribuir para o conhecimento da soroprevalência da sífilis, nos grupos vulneráveis das regiões nacionais e internacionais, favorece o estabelecimento de fluxogramas e indicadores de avaliação e monitoramento de estratégias para acompanhamento da infecção em população para além das instituições de saúde ${ }^{27,28,33}$.

Estudos, cujo objetivo foi relacionar a sífilis com outras infecções sexualmente transmissíveis, como HIV, Hepatite C e gonorreia somam $4(17,3 \%)^{25-27,30}$. É preciso ponderar que a prevalência elevada de infecções sexualmente transmissíveis entre as mulheres, nos países em desenvolvimento, tem gerado preocupação e exigido a expansão dos métodos de prevenção e detecção para manutenção da saúde, em concomitância com o controle das infecções oportunistas, não só em nível nacional, mas no âmbito internacional.

Vale registrar que reflexões sobre o público masculino foram identificadas em apenas um artigo, destacando a saúde sexual como um portal da saúde do homem ${ }^{31}$. A falta de pesquisas que abarquem a saúde masculina e a sífilis chama atenção, principalmente, pela expansão desta infecção, exprimindo a necessidade de promover iniciativas de saúde no sentido de acolher esta clientela $31,35,36$

A respeito dos participantes das pesquisas revisadas, $11(47,82 \%)$ fizeram estudos com seres humanos e contextualizaram as dimensões do ser feminino, seja no período gravídico puerperal, na situação de vulnerabilidade ou em idade fértil ${ }^{9,10,16-18,20-25}$. Todavia ao refletir sobre o perfil socioeconômico, verificam-se mulheres jovens, com baixa escolaridade e baixa condição socioeconômica, que convivem em relações desiguais de gênero. Pondera-se que as históricas desigualdades de poder entre homens e mulheres devem ser consideradas como um dos determinantes da saúde na formulação das políticas públicas, principalmente na erradicação de infecções como a sífilis ${ }^{35}$.

Segundo a variável delineamento da pesquisa, 21(91,3\%) estudos foram descritivos (não experimentais) com observação de resultados terapêuticos e evolução clínica ${ }^{9-18,20,22-31}$, destes, 3(13,2\%) correspondem a publicações com aspecto sócio-histórico-cultural ${ }^{16-18}$. Encontrou-se $1(4,3 \%)$ artigo com metodologia baseada no estudo de caso ${ }^{21}$ e $1(4,3 \%)$ publicação que utilizou como método de pesquisa a revisão integrativa da literatura ${ }^{19}$. Porém, somente $3(13,2 \%)$ mencionaram os preceitos éticos ${ }^{16,18,30}$, com o registro da aprovação do comitê de ética em pesquisa. Constatou-se que $7(30,4 \%)$ estudos provêm de outros países ou foram realizados antes das normatizações da Comissão Nacional de Ética em Pesquisa17,20-22,24,26,27. 
Os desenhos de estudos de natureza descritiva (estudos não experimentais) não são considerados fortes evidências para aplicação clínica. Isso incita a contribuir para o fortalecimento dos grupos de colaboração Cochrane, com centros em diferentes países que oferecem informações atuais e de alta qualidade e que têm como meta elaborar e disseminar as revisões sistemáticas que retratam a eficácia de intervenções na área da saúde ${ }^{37}$.

É imperioso sublinhar que, dos $21(91,3 \%)$ estudos com abordagem descritiva (não experimentais) ${ }^{9-18,20,22-31}$, somente $2(8,9 \%)$ abordaram os cuidados de enfermagem ao valorizar a voz da mulher com sífilis, compreendendo que o ser humano necessita se perceber como um agente potencial e capaz de escolhas responsáveis quando estimulados para adoção de hábitos de vida seguros e saudáveis ${ }^{16,18}$.

Tomando por base esses dados, pode-se realçar a importância da elaboração de pesquisas socioculturais. A vulnerabilidade ao adoecer, principalmente por infecção sexualmente transmissível, pode ser potencializada ou minimizada em circunstâncias de vidas individuais, nas quais cada um dos parceiros alcança certa proporção de acordo com seu contexto social, cultural e econômico ${ }^{38,39}$.

Quanto à variável nível de evidência, os resultados encontrados indicam que a maioria das publicações, desta revisão integrativa, possui nível de evidencia científica $2 C-21(91,3 \%)^{9-18,20,22-31} ; 1(4,3 \%)$ com nível de evidencia científica $4^{21}$; e $1(4,3 \%)$ publicação com nível de evidência científica $5^{19}$. O nível $2 \mathrm{C}$, disponível na maioria dos artigos revisados, indica evidência científica moderada, mas não permite fazer recomendações a favor ou contra a intervenção clínica preventiva, sendo necessário explorar critérios individuais, tais como população, etiologia e prognóstico para a melhor decisão interventiva. Já os artigos com níveis 4 e 5 são analisados como evidências científicas insuficientes, não havendo critérios satisfatórios para fazer uma recomendação em saúde.

Assim, é prudente reconhecer que os estudos em pauta não aplicaram métodos que conduzam à síntese das melhores evidências científicas, não havendo justificativa para a generalização das recomendações delineadas, contudo, as reflexões e propostas parecem ter sido pertinentes para os estudos envolvendo a sífilis na mulher e no homem. Pressupõe-se que o conhecimento da classificação de evidências científicas dos artigos, recuperados nesta revisão integrativa, proporciona subsídios para auxiliar os profissionais de saúde a realizarem uma avaliação crítica de resultados oriundos de pesquisas e, consequentemente, contribui para a tomada de decisão sobre a incorporação das evidências à prática clínica relacionada a sífilis ${ }^{6,9-31}$.

Em relação a variável periódicos, $12(52,1 \%)$ artigos foram publicados em revistas classificadas como Qualis $A 2^{9,10,12-15,18,19,22,24,30,31} ; 4(17,3 \%)$ em revistas avaliadas com Qualis $B 1^{11,17,25,27}$; os demais foram publicados em periódicos com Qualis B2, B3 e B4 16,20,21,23,26,28,29. Ponderar a classificação Qualis é considerar aspectos relevantes como: qualidade dos artigos, do corpo editorial e dos consultores, critérios de arbitragem dos textos, natureza do órgão publicador e no que diz respeito a estratégias que visam assegurar a excelência da produção científica divulgada.

No que concerne à indexação dos periódicos, 16(69,3\%) estão indexadas nas bases de dados nacionais e internacionais ${ }^{9-15,17-19,22,24,25,27,30,31}$. Informações como estas são necessárias uma vez que quanto maior o número de bases de dados indexadas, maior será o padrão da produtividade e a difusão indireta da revista e do seu conteúdo 32,34 .

Não foram encontradas referências relativas ao financiamento nas publicações. Isto talvez seja reflexo das rápidas mudanças no cenário dos subsídios às pesquisas. Cabe analisar criticamente estas mudanças de distribuição dos recursos destinados à pesquisa, principalmente em função dos mecanismos tradicionais de financiamento dos projetos de pequena escala.

\section{CONCLUSÃO}

A maioria dos artigos foi classificada no nível 2C, considerado evidência científica moderada, não se justificando a generalização de recomendações, mas pondera-se que as reflexões e propostas parecem ter sido pertinentes para os 23 estudos envolvendo a sífilis na mulher e no homem.

A escassez de pesquisas que abarquem a saúde masculina e a sífilis chama atenção sobre a expansão dessa infecção sexualmente transmissível e de cura reconhecida desde a descoberta da penicilina na década de 40, exprimindo a necessidade de promover iniciativas de educação em saúde no sentido de acolher essa clientela, esclarecendo as formas de transmissão, diagnóstico, tratamento concomitante ao autoconhecimento, como o uso dos preservativos para a prática do sexo vaginal, anal e oral.

Constatou-se também que a temática sífilis é muito explorada em assuntos que abrangem o pré-natal, obstetrícia ou a transmissão vertical, havendo um hiato de conhecimentos científicos voltados para a temática sífilis fora do ciclo gravídico-puerperal. 
Este artigo não se esgota com estas considerações, porém, traz à tona as imensas possibilidades de estudos multicêntricos nas diversas regiões do Brasil e do mundo, apontando para a necessidade de continuidade das pesquisas sobre o avanço na prevenção da sífilis.

\section{REFERÊNCIAS}

1. Nascimento MI, Cunha AA, Guimarães EV, Alvarez FS, Oliveira SRSM, Villas Bôas EL. Pregnancies complicated by maternal syphilis and fetal death. Rev. bras. ginecol. obstet. 2014 [cited 2018 Feb 18]; 34(2):56-62. Available from: http://www.scielo.br/pdf/rbgo/v34n2/a03v34n2.pdf

2. Figueiró EAF, Freire SSA, Souza BA, Aguena GS, Maedo CM. Sífilis e gestação: estudo comparativo de dois períodos (2006 e 2011) em população de puérperas. DST j. bras. doenças sex. transm. 2012 [cited 2018 Feb 18]; 24(1):32-7. Available from: http://www.dst.uff.br/revista24-1-2012/9.Sifilis\%20e\%20Gestacao\%20Estudo\%20Comparativo\%20de\%20Dois\%20Periodos.pdf

3. Magalhães DMS, Kawaguchi IAL, Dias A, Calderon IMP. Maternal and congenital syphilis: a persistent challenge. Cad. Saúde Pública (Online). 2013 [cited 2018 Mar 18]; 29(6):1109-20. Available from: http://www.scielo.br/pdf/csp/v29n6/a08v29n6.pdf

4. Knauth DR, Couto MT, Figueiredo WS. The standpoint of professionals on the presence and demands of men on the healthcare services: perspectives for the analysis of the implementation of the Comprehensive Healthcare Policy for Men. Ciênc. saúde coletiva (Online). 2012 [cited 2018 Jul 16]; 17(10):2617-26. Available from: http://www.scielo.br/pdf/csc/v17n10/11.pdf

5. Cooper HM. The integrative research review: a systematic approach. Beverly Hills (CA): Sage Pulications; 1984.

6. Sackett DL, Rosenberg WMC. On the need for evidence-based medicine. Am. j. public health. 2009 [cited 2018 Apr 16]. 17(3):330-4. DOI: https://doi.org/10.1093/oxfordjournals.pubmed.a043127

7. Soares CB, Hoga LAK, Peduzzi M, Sangaleti C, Yonekura T, Silva DRAD. Integrative review: concepts and methods used in nursing. Esc. Enferm. USP. 2014 [cited 2018 Jul 16]; 48(2):335-45. DOI: http://dx.doi.org/10.1590/S00806234201400002000020

8. Costa LHR, Coelho ECA. Nursing and sexuality: Integrative review of papers published by the Latin-American Journal of Nursing and Brazilian Journal of Nursing. Rev. latinoam. enferm. (Online). 2011 [cited 2018 Feb 10]; 19(3):631-9. Available from: http://www.scielo.br/pdf/rlae/v19n3/pt_24.pdf

9. Vaz AJ, Guerra EM, Ferratto LMC, Toledo LAS, Azevedo RS. Sorologia positiva para sífilis, toxoplasmose e doença de chagas em gestantes de primeira consulta em centros de saúde de área metropolitana, Brasil. Rev. saúde pública (Online). 1990 [cited 2018 Mar 10]; 24(5):373-9. Available from: http://www.scielo.br/pdf/rsp/v24n5/04.pdf

10. Fernandes RCSC, Fernandes PGCC, Nakata TY. Evaluation of congenital syphilis cases at the maternity of the Hospital da Sociedade Portuguesa de Beneficência de Campos, RJ. DST j. bras. doenças sex. transm. 2007 [cited 2018 Apr 11]; 19(3):157-61. Available from: http://pesquisa.bvsalud.org/portal/resource/pt/lil-530220

11. Saraceni V, Domingues RMSM, Vellozo V, Lauria LM, Dias MAB, Ratto KMN. Surveillance of syphilis in pregnancy. Epidemiol. serv. saúde. 2007 [cited 2018 May 11]; 16(2):103-11. Available from: http://scielo.iec.gov.br/pdf/ess/v16n2/v16n2a05.pdf

12. Saraceni V, Guimarães MHFS, Theme MM, Leal MC. Perinatal mortality due to congenital syphilis: a quality-of-care indicator for women's and children's healthcare. Cad. Saúde Pública (Online). 2005 [cited 2018 Jul 18]; 21(4):1244-50. Available from: http://www.scielo.br/pdf/csp/v21n4/27.pdf

13. Saraceni V, Vellozo V, Leal MC, Hartz ZMA. Reliability of the national disease surveillance data system using campaigns for the elimination of congenital syphilis in the city of Rio de Janeiro. Rev. bras. epidemiol. 2005 [cited 2018 Jul 18]; 8(4):419-24. Available from: http://www.scielo.br/pdf/rbepid/v8n4/08.pdf

14. Saraceni V, Vellozo VRO, Leal MC, Hartz ZMA. Congenital syphilis campaigns evaluation in the City of Rio de Janeiro based on a theoretical logical model. Rev. Bras. Saúde Mater. Infant. (Online). 2005 [cited 2018 Jul 18]; 5(1):33-41. Available from: http://www.scielo.br/pdf/rbsmi/v5s1/27839.pdf

15. Saraceni V, Leal MC. Evaluation of the effectiveness of the congenital syphilis elimination campaigns on reducing the perinatal morbidity and mortality. Rio de Janeiro, 1999-2000. Cad. Saúde Pública (Online). 2003 [cited 2018 Mar 01]; 19(5):1341-9. Available from: http://www.scielo.br/pdf/csp/v19n5/17806.pdf

16. Silva AM, Sousa JC, Albuquerque S, Moreira CA, Martins MC. Feelings of pregnant women diagnosed with syphilis. Rev. enferm. UFPI. 2015 [cited 2018 Jun 02]; 4(2):84-91. Available from: http://www.ojs.ufpi.br/index.php/reufpi/article/view/3336/pdf

17. Fitzgerald DW, Behets F, Caliendo A, Roberfroid D, Lucet C, Fitzgerald JW. Economic hardship and sexually transmitted diseases in Haiti's rural artibonite valley. Am. j. trop. med. hyg. 2000 [cited 2018 Jul 21]; 62(4):496-501. Available from: https://www.ncbi.nlm.nih.gov/pubmed/11220766

18. Silva LR, Santos RS. What the mothers know and feel on syphilis congenital: an exploitation study and its implications for the nursing practice. Esc. Anna Nery Rev. Enferm. 2014 [cited 2018 Jul 22]; 8(3):393-401. Available from: https://www.redalyc.org/pdf/1277/127718062010.pdf

19. Walker GJA. Antibiotics for syphilis diagnosed during pregnancy. Cochrane database syst. rev. (online). 2006 [cited 2018 Jul 22]; 4(3):11-43. DOI: https://doi.org/10.1002/14651858.CD001143

20. Folgosa E, Osman NB, Gonzalez C, Hägerstrand I, Bergström S, Ljungh A. Syphilis seroprevalence among pregnant women and its role as a risk factor for stillbirth in Maputo, Mozambique. Genitourin. med. 1996 [cited 2018 Aug 03]; 72(5):339-42. Available from: https://pdfs.semanticscholar.org/77b0/f24022aed2091e313fb616d1946fcaa841d9.pdf

21. Gouvea TVD, Faria PFM, Nascimento AVS, Firmo FHC, Santos DDG. Sífilis na Gravidez: Relato de (Des)Caso. Rev. flum. med. cir. espec. 2012 [cited 2018 Aug 03]; 77(2):36-9. Available from: http://amf.org.br/site/docs/rfm/2012/11S\%C3\%ADfilis\%20na\%20Gravidez.pdf 
22. Hoek A, Yuliang F, Dukers NHTM, Zhiheng C, Jiangting F, Lina Z. High prevalence of syphilis and other sexually transmitted diseases among sex workers in China: potential for fast spread of HIV. AIDS care. 2001 [cited 2018 Aug 04]; 15(7):53-9. Available from: https://pdfs.semanticscholar.org/5bb3/26083811ca0e910b9ee1e66029228c75610d.pdf

23. Pistarin JAP, Brancacio BL, Buzo CF, Alves BA. Seroprevalence of hiv, syphilis, and hepatites $B$ and $C$ among women confined at Centro de Ressocialização Feminino of Rio Claro, São Paulo. Rev. Inst. Adolfo Lutz. 2006 [cited 2018 Aug 04]; 65(2):133-6. Available from: http://periodicos.ses.sp.bvs.br/pdf/rial/v65n2/v65n2a12.pdf

24. Camejo M, Mata G, Díaz M. Prevalence of hepatitis B, hepatitis C and syphilis in female sex workers in Venezuela. Rev. saúde pública (Online). 2003 [cited 2018 Aug 05]; 37(3):339-44. Available from: http://www.scielo.br/pdf/rsp/v37n3/15862.pdf

25. Pando MA, Reynaga E, Coloccini RS, Fermepín MR, Kochel T, Montano SM. Prevalence of HIV infection and Treponema pallidum in Argentine female sex workers. Rev. panam. salud pública. 2011 [cited 2018 Aug 15]; 30(4):303-8. Available from: https://www.scielosp.org/article/rpsp/2011.v30n4/303-308/es

26. Long CM, Klausner JD, Leon S, Jones FR, Giron M, Cuadros J. Syphilis treatment and HIV infection in a population-based study of persons at high risk for sexually transmitted disease/HIV infection in Lima, Peru. Sex. transm. dis. 2006 [cited 2018 Aug 15]; 33(3):151-5. Available from: https://journals.Iww.com/stdjournal/Fulltext/2006/03000/Syphilis_Treatment_and_HIV_Infection_in_a.6.aspx

27. Hamers FF, Peterman TA, Zaidi AA, Ransom RL, Wroten JE, Witte JJ. Syphilis and Gonorrhea in Miami: Similar Clustering, Different Trends. Am. J. public. health. 1995 [cited 2018 Aug 15]; 85(8):1004-8. DOI:

https://doi.org/10.2105/AJPH.85.8_Pt_1.1104

28. Kuklová I, Velčevský P, Kojanová M. Syphilis among STD clinic patients in Prague in 2009. Cent. eur. j. public health. 2011 [cited 2018 Aug 15]; 19(2):84-90. Available from: https://pdfs.semanticscholar.org/593c/37da0f78b99f9be51364ed7349f0ce199411.pdf

29. Marra CM, Maxwell CL, Smith SL, Lukehart SA, Rompalo AM, Eaton M. Cerebrospinal fluid abnormalities in patients with syphilis: association with clinical and laboratory features. J. infect. dis. 2011 [cited 2018 Aug 16]; 189(3):369-76. DOI: https://doi.org/10.1086/381227

30. Ramos VM, Figueiredo EN, Succi RCM. Barriers to control syphilis and HIV vertical transmission in the health care system in the city of Sao Paulo. Rev. bras. epidemiol. 2014 [cited 2018 Aug 15]; 17(4):887-98. DOI: https://doi.org/10.1590/18094503201400040008

31. Pinto VM, Tancredi MV, Alencar HDR, Camolesi E, Holcman MM, Grecco JP. Prevalence of Syphilis and associated factors in homeless people of Sao Paulo, Brazil, using a Rapid Test. Rev. bras. epidemiol. 2014 [cited 2018 Aug 15]; 17(2):341-54. DOI: http://dx.doi.org/10.1590/1809-4503201400020005ENG

32. Oelkea ND; Lima MADS; Costa AMA. Knowledge translation: translating research into policy and practice. Rev. gaúch. enferm. 2015 [cited 2018 Aug 15]; 36(3):113-7. DOI: https://doi.org/10.1590/1983-1447.2015.03.55036

33. Rohden F. Accessed through sex: the medicalization of male sexuality at two different moments. Ciênc. saúde coletiva (Online). 2012 [cited 2018 Aug 16]; 17(10):2645-54. Available from: http://www.scielo.br/pdf/csc/v17n10/14.pdf

34. Erdmann AL, Fernandes JD, Teixeira GA. Panorama da educação em enfermagem no Brasil: graduação e pós-graduação. Enferm. foco (Brasília). 2012 [cited 2018 Aug 16]; 2(3):89-93. Available from: http://revista.cofen.gov.br/index.php/enfermagem/article/view/91/76

35. Lima M, Schraiber LB. Violence and Other Gender Vulnerabilities and Women Living with HIV/Aids. Temas psicol. (Online). 2013 [cited 2018 Aug 18]; 21(3):947-60. DOI: http://doi.org/10.9788/TP2013.1-EE09PT

36. Corrêa ACDP, Mozer IT. Managing the process of implementing men's health policy. Rev. enferm. UERJ. 2016 [cited 2018 Aug 18]; 24(1),e9483. DOI: https://dox.doi.org/10.12957/reuerj.2016.9483

37. Carvalho APV, Silva V, Grande AJ. Avaliação do risco de viés de ensaios clínicos randomizados pela ferramenta da colaboração Cochrane. Diagn. tratamento. 2013 [cited 2018 Aug 18]; 18(1):38-44. Available from: http://files.bvs.br/upload/S/14139979/2013/v18n1/a3444.pdf

38. Nicolau AIO, Ribeiro SG, Lessa PRA, Monte AS, Bernardo EBR, Pinheiro AKB. Knowledge, attitude and practices regarding condom use among women prisoners: the prevention of STD/HIV in the prison setting. Esc. Enferm. USP. 2012 [cited 2018 Aug 15]; 46(3):711-9. Available from: http://www.scielo.br/pdf/reeusp/v46n3/25.pdf

39. MachadoYY, Nogueira VPF, Oliveira DC, Gomes AMT. Health personnel's social representations of HIV/AIDS: a structural analysis. Rev. enferm. UERJ. 2016 [cited 2018 Aug 15]; 24(1):e14463. DOI: https://doi.org/10.12957/reuerj.2016.14463 